\title{
Cluster-Based Ranging for Accurate Localization in Wireless Sensor Networks
}

\author{
Simone Sergi, ${ }^{1}$ Fabrizio Pancaldi, ${ }^{2}$ and Giorgio M. Vitetta ${ }^{1}$ \\ ${ }^{1}$ Department of Information Engeneering, University of Modena e Reggio Emilia, Italy \\ ${ }^{2}$ Department of Science and Methods for Engeneering, University of Modena e Reggio Emilia, Italy
}

Correspondence should be addressed to Giorgio M. Vitetta, giorgio.vitetta@unimore.it

Received 13 November 2009; Revised 21 May 2010; Accepted 1 June 2010

Academic Editor: Ronald Raulefs

Copyright ( 2010 Simone Sergi et al. This is an open access article distributed under the Creative Commons Attribution License, which permits unrestricted use, distribution, and reproduction in any medium, provided the original work is properly cited.

\begin{abstract}
A novel ranging technique based on received signal strength (RSS) and suitable to indoor scenarios is illustrated. In the proposed technique, multiple power measurements, associated with the signals radiated by a cluster of nodes surrounding a given target, are jointly processed to improve the quality of RSS-based estimation of the distance between the target and an anchor. Specific algorithms for the generation of a cluster and for the acquisition of power measurements are described. Simulation results show that, when used in indoor positioning systems, the proposed ranging technique is substantially more accurate than noncooperative strategies. In addition, it allows to concentrate significant processing tasks in a limited number of fixed anchors, so reducing maintenance costs and making it possible to adopt cheap and simple portable wireless nodes.
\end{abstract}

\section{Introduction}

Wireless sensor networks for accurate localization of people and objects in indoor environments represent a fundamental tool for the provisioning of high-level services in the fields of management and logistics. Despite this, currently the pervasive diffusion of such networks is prevented by their large complexity and maintenance costs. This is mainly due to the fact that, in indoor scenarios, accurate localization in the presence of severe multipath fading usually requires a large number of fixed sensing nodes (called anchors) with a known location [1] and the use of complicated signal processing algorithms for distributed distance estimation [2]. It is also important to note that localization algorithms are often designed under the assumption that accurate pointto-point distance measurements between the target node (i.e., that whose location needs to be identified) and a set of anchors are available [3-7]. Unluckily, the first hypothesis can be deemed realistic only when there is a line of sight (LOS) path between the given target node and the anchors involved in distance measurements, so that the first echo (i.e., the one with the shortest time of arrival) can be separated from all the following ones, originating from nonline of sight (NLOS) paths, via proper signal processing algorithms
[8]. However, the accuracy achievable in such measurements reduces noticeably in indoor environments, since, generally speaking, they are characterized by significant obstacles to signal propagation and, frequently, by NLOS links [9].

Recently, an increasing attention has been paid to the use of ultrawide band (UWB) transmission techniques [10] for radio localization in indoor scenarios. This interest is motivated by the appreciable time resolution originating from the extremely wide bandwidth of UWB signalling formats [11]. This means that multiple echoes of UWB signals can be accurately resolved by sensing nodes, so that, in principle, the quality of ranging estimation can be substantially improved.

In this work, a new solution to indoor localization based on a UWB wireless sensor network is illustrated. The derivation of the proposed technique relies on the use of low complexity ranging estimation methods based on received signal strength (RSS) $[2,12]$, on some considerations about the statistical properties of UWB channels, and the use of multiple (i.e., of a cluster of) transmitting nodes, placed in different points of the considered environment, to refine the distance measurement of a target from a given anchor. These design choices can be motivated as follows: (1) measuring RSS is simple and can be easily accomplished 
even by receivers which have not been explicitly designed to perform distance estimation (so avoiding the additional costs of deploying ad hoc hardware); (2) recent studies about statistical modeling of indoor UWB channels (e.g., see [1315]) provide accurate information about the power decay model and the distribution of multipath components; (3) RSS indirectly exploits the very wide bandwidth of UWB signals, since such signals exhibit little small scale fading when compared to narrowband signals [16]; and (4) the use of multiple low-cost transmitting nodes allows to mitigate the effects of fading, resulting in improved accuracy. As far as the last point is concerned, it is important to note that the adopted solution exploits a sort of cooperative diversity, since, in the range measurement procedure, spatially separated nodes cooperate, emitting localization signals, which travel, however, through distinct wireless channels. It is also worth pointing out that, as far as we know, until now statistical modeling of UWB channels has been exploited to improve the overall performance of localization (e.g., see $[4,17]$ ), but not the quality of ranging measurements.

The remaining part of the paper is organized as follow. The use of a cluster of nodes for improving ranging measurements is motivated in detail in Section 2, whereas algorithms for ranging estimation based on RSS and on the use of a cluster of nodes, and methods for cluster generation are derived in Section 3. In Section 4, the problem of acquiring independent RSS measurements is tackled, whereas in Section 5 some simulation results evidencing the enhancement in ranging accuracy provided by the proposed algorithms are analyzed. Finally, Section 6 offers some conclusions.

\section{Cooperative Ranging Based on a Cluster of Nodes}

In a wireless sensor network for indoor localization, the scope of each anchor is estimating its distance from a given target node on the basis of the RSS associated with the digital signal radiated by the node itself. In a static scenario affected by multipath fading, the average power $R$ associated with the useful component of the signal received at a given anchor can be expressed as [18]

$$
R=P_{0}-(\mathrm{PL}+\mathrm{LSF}+\mathrm{SSF})
$$

where $P_{0}$ is a reference power level, and PL, LSF, and SSF denote the exponential path loss, the large scale fading, and the small scale fading (all expressed in $\mathrm{dBm}$ ) affecting the communication channel, respectively. In the following an NLOS condition is assumed for signal propagation, so that LSF and SSF can be represented as zero mean random parameters. In particular, LSF is modelled as a zero mean Gaussian random variable whose variance is described, in turn, by a Gaussian distribution characterized by known parameters; such parameters depend on the characteristics of the building, in which signal propagation occurs [14]. In addition, the statistical behavior of SSF is described by a Nakagami distribution with an $m$-factor $m \geq 1 / 2$ [15]. As far as the path loss PL is concerned, following
$[14,15]$, an exponential power decay is considered; its path gain exponent, however, is modeled as a Gaussian random variable having constant variance and known mean, both depending on the macroscopic characteristics of the considered indoor environment.

In the absence of fading, we have that SFF $=\mathrm{LSF}=0$ in (1); then, the distance of the target node from a selected anchor can be perfectly estimated from $R$ if the power level $P_{0}$ and the mathematical model of the PL are completely known. Unluckily, the presence of fading introduces an uncertainty in ranging measurements based on (1). To mitigate fading effects, we propose to exploit, in distance estimation, all the RSSs associated with the digital signals radiated by a spatial cluster of nodes, as illustrated in Figure 1. This cluster has the following properties: (a) it consists of a subset of $N$ radiating nodes selected in a pool of $N_{T}$ nodes; (b) it includes the target node; (c) the signals transmitted by its nodes, as well as the signals transmitted by all the other nodes in the network, are mutually orthogonal in a specific domain (e.g., in the time or in the frequency domain) and can be separated perfectly by the anchor. Then, if $a_{n}$ denotes the $n$th node of a given cluster $C$ (i.e., $C$ is the set $\left\{a_{n}, n=1,2, \ldots, N\right\}$ ), the average power $R_{\mathrm{av}}$ captured by the anchor is given by (see (1))

$$
\begin{aligned}
R_{\mathrm{av}} & \triangleq \frac{1}{N} \sum_{n=1}^{N} R_{n} \\
& =P_{0}-\left(\frac{1}{N} \sum_{n=1}^{N} \mathrm{PL}_{n}+\frac{1}{N} \sum_{n=1}^{N} \mathrm{LSF}_{n}+\frac{1}{N} \sum_{n=1}^{N} \mathrm{SSF}_{n}\right),
\end{aligned}
$$

where $R_{n}$ denotes the average power received from $a_{n}$, whereas $\mathrm{PL}_{n}, \mathrm{LSF}_{n}$, and $\mathrm{SSF}_{n}$ are the exponential path loss, the large scale fading, and the small scale fading affecting the communication channel between the anchor and $a_{n}$. The path loss $\mathrm{PL}_{n}$ can be expressed as

$$
\mathrm{PL}_{n}=\mathrm{PL}_{\text {mean }}+\Delta \mathrm{PL}_{n}
$$

for $n=1,2, \ldots, N$, where $\mathrm{PL}_{\text {mean }}$ represents the average path loss affecting the cluster nodes, whereas $\Delta \mathrm{PL}_{n}$ denotes the offset of $\mathrm{PL}_{n}$ from $\mathrm{PL}_{\text {mean }}$. Intuitively, $\mathrm{PL}_{\text {mean }}$ accounts for the loss associated with the separation between the cluster and the anchor, whereas $\Delta \mathrm{PL}_{n}$ can be related to the displacement of the nodes within the cluster, as shown in Figure 2. It is also worth noting that, generally speaking, the value of the path loss exponent depends on the propagation link [14, 15], so that, in principle, it can take on different values for distinct nodes of the same cluster; however, modelling errors concerning this parameter in the evaluation of path loss can be incorporated in the $\left\{\Delta \mathrm{PL}_{n}\right\}$ terms.

Given (3), (2) can be rewritten as

$$
R_{\mathrm{av}}=P_{0}-\left(\mathrm{PL}_{\text {mean }}+\Delta \mathrm{PL}+\frac{1}{N} \sum_{n=1}^{N} \mathrm{LSF}_{n}+\frac{1}{N} \sum_{n=1}^{N} \mathrm{SSF}_{n}\right) \text {, }
$$

where

$$
\Delta \mathrm{PL} \triangleq \frac{1}{N} \sum_{n=1}^{N} \Delta \mathrm{PL}(n)
$$




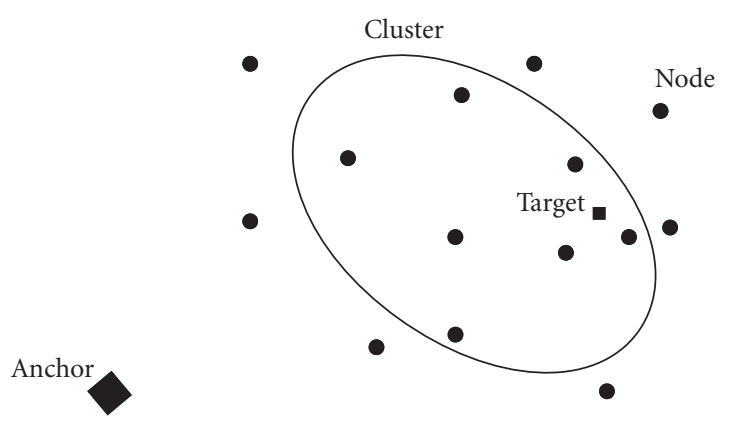

Figure 1: Set-up of the analysed system.

If ranging estimation of the selected anchor is based on the quantity $R_{\mathrm{av}}$ (2) instead of $R$ (1), a larger accuracy can be achieved thanks to the fading mitigation effect originating from the use of multiple contributions. This can be easily proved if the indoor scenario is characterized by a rich scattering, so that the channels between distinct nodes and the selected anchor can be deemed statistically independent. Then, the random variables $\left\{\mathrm{LSF}_{n}\right\}$ and $\left\{\mathrm{SSF}_{n}\right\}$ are mutually independent and, if the radiating nodes are identical and the associated channels are statistically equivalent, can be considered identically distributed. Since the variance of the fading terms $\left\{\mathrm{LSF}_{n}\right\}$ and $\left\{\mathrm{SSF}_{n}\right\}$ is finite, if $N$ is large enough, the central limit theorem [19] can be applied, and each of the terms $\sum_{n=1}^{N} \mathrm{LSF}_{n}$ and $\sum_{n=1}^{N} \mathrm{SSF}_{n}$ in (4) can be modelled as zero mean Gaussian random variable with a variance tending to 0 as $N$ increases; in other words, the ranging error component due to multipath fading can be substantially reduced if the number $N$ of (cooperating) cluster nodes is large enough. Note, however, that the achievable accuracy can be limited by the term $\triangle \mathrm{PL}$ (5), representing the residual error due to the spatial spread of the selected cluster. For instance, if the nodes of the cluster are placed in a regular fashion along a circumference and the anchor is placed in its center, the term $\triangle \mathrm{PL}$ will be equal to zero, independently of the distance between the nodes and the anchor, even if this increases to infinity (see the example of Sestion 5.1 for a deeper insight). This means that, in principle, the term $\Delta \mathrm{PL}$ is not minimized if a spatially dense cluster is selected, but if the nodes forming the cluster are enrolled according to a clever strategy.

It is also important to point out that the approach we propose for improving the quality of ranging measurements can provide various practical advantages; some of them are listed below.

(i) If the proposed method can achieve a better accuracy in NLOS conditions then that offered by classic RSSbased ranging techniques, the application level can be simplified, since most of the computational burden is concentrated at lower system layers.

(ii) The employed radio nodes can be extremely simple (and, hence, cheap), since most of signal processing for localization is accomplished by a small number of fixed anchors. In fact, As pointed out in the following,

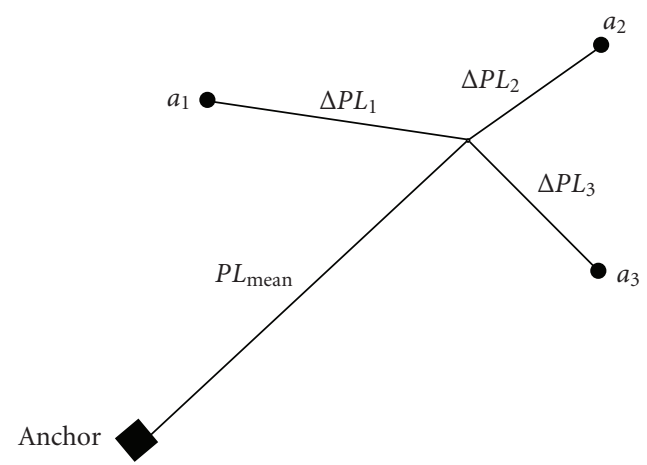

FIGURE 2: Decomposition of the path loss terms of a cluster of nodes in a common cluster distance-related term $\left(\mathrm{PL}_{\text {mean }}\right)$ and a set of distance errors $\left(\Delta \mathrm{PL}_{n}\right)$.

each node is only expected to respond to a query with a certain (and precise) power level.

(iii) System performance can be improved putting dummy nodes in the environment; these have the only scope of increasing node density.

The adoption of the proposed solution, however, raises some important technical issues that need to be carefully addressed. In particular, we note that

(i) a proper strategy for the generation of a cluster is needed to identify the nodes optimising performance, that is, the overall ranging accuracy,

(ii) the impact of node density over the system performance should be analyzed. In fact, on the one hand, if the cluster covers a small area, the residual error $\triangle \mathrm{PL}$ is negligible with respect to the mean distance (which can be identified as the target distance) associated with $\mathrm{PL}_{\text {mean }}$; however, it is likely that in this situation the fading affecting distinct nodes exhibits a substantial correlation, so that the effectiveness of the proposed strategy can be appreciably affected. On the other hand, if the cluster size is significant, it is not difficult to show that the ranging error due to the fading terms $\left\{\mathrm{LSF}_{n}\right\}$ and $\left\{\mathrm{SSF}_{n}\right\}$ decreases as $1 / N^{2}$ only; the estimation accuracy, however, can get worse because of $\triangle \mathrm{PL}$, that is, of the spread in node locations with respect to cluster center.

The first problem can be solved using proper algorithms, as illustrated in Section 3. Unluckily, the second problem does not lend itself to a simple mathematical analysis. For this reason, its relevance has been assessed via computer simulations; some results are illustrated and commented in Section 5 .

\section{Cluster Selection Strategies}

In this Section, two different strategies for the selection of the wireless nodes forming a cluster for a given target node in a static indoor scenario are proposed. Then, the problem 
of distance estimation based on a set of data acquired from a cluster of nodes is analyzed.

Before analysing the proposed strategies in detail, it is worth noting that any strategy for cluster selection should aim at identifying a group of nodes (in the pool of available ones) in a way that the fading effects in the measurement of $R_{\mathrm{av}}$ (4) are mitigated and that, at the same time, the amplitude of the term $\triangle \mathrm{PL}$ (5) is not enhanced. For this reason, the problem of selecting an optimal cluster $\widehat{C}$ could be formulated as

$$
\widehat{C}=\arg \min _{\widetilde{C}, N_{\widetilde{C}}}\left|R_{\mathrm{av}}\left(\widetilde{C}, N_{\widetilde{C}}\right)-\left(P_{0}-\mathrm{PL}_{\text {mean }}\left(\widetilde{C}, N_{\widetilde{C}}\right)\right)\right|,
$$

where the dependence of $R_{\mathrm{av}}$ and $\mathrm{PL}_{\text {mean }}$ on the trial cluster $\widetilde{C}$ and on its size $N=N_{\widetilde{C}}$ is explicitly indicated. Note that

(i) the cluster optimization procedure expressed by (6) involves not only the selection of a specific set of nodes $\widetilde{C}$, but also that of its size $N_{\tilde{C}}$. If $N_{\tilde{C}}$ is large, fading is mitigated, but the effects of the offset $\Delta \mathrm{PL}$ become significant,

(ii) if $N_{T}$ denotes the overall number of nodes in the environment managed by the anchor, the number of unordered collections of $N_{\widetilde{C}}$ nodes extracted from a set of $N_{T}$ nodes is given by $\left(\begin{array}{c}N_{T} \\ N_{\tilde{C}}\end{array}\right)=N_{T} ! /\left(N_{\tilde{C}} !\left(N_{T}-\right.\right.$ $\left.N_{\widetilde{C}}\right)$ !); this quantity (and, consequently, the computational complexity of the problem (6)) grows quickly as $N_{\widetilde{C}}$ increases.

The last comment motivates the investigation of suboptimal strategies leading to a good accuracy in distance estimation at the price of a reasonable complexity. In the following, Paragraphs we show that such strategies can be developed adopting a heuristic approach to the problem of cluster selection. Our strategies operate in a centralized fashion, since cluster selection is accomplished at the anchor. For this reason, in the cluster selection procedure, each involved node is only expected to generate a signature signal in response to a radio frequency query from the anchor. In the following, however, we do not tackle the problems of identifying each node (and, in particular, of looking for the target node in the cluster) and of managing the access to a shared radio medium. In fact, we simply assume that the anchor is able to identify each node, exploiting, for instance, an unambiguous identification code wired in the hardware of each node, like in RFID tags [20]. Moreover, the response signal is expected to be transmitted at a certain (and precise) power level, so that active RFIDs [20] could represent a good technical choice in a low-cost node design.

Finally, we note that, as already mentioned in the previous Paragraph, the identification of the optimal cluster $\hat{C}$ relies on the availability of several independent RSS measurements for each node of the trial clusters $\widetilde{C}$; two different strategies for the acquisition of such data are illustrated in Section 4. Here we consider a static scenario, in other wongs we assume that negligible changes occur in the measurement scenario during the interval in which RSS data are acquired. If such an interval is very short, then the solutions we propose can be adopted for portable indoor applications.

3.1. Cluster Selection Based on the Minimization of a Power Spread. The first procedure for cluster selection evolves through the following steps:

(1) $N_{P}$ independent power measurements $\left\{R_{n}(m), m=\right.$ $\left.1,2, \ldots, N_{P}\right\}$ are acquired for the node $a_{n}$ (with $n=1$, $2, \ldots, N_{T}$ ) reached by a query signal from the anchor;

(2) a trial cluster $\widetilde{C}$, having fixed size $D$ and including the target node, is selected;

(3) for $m=1,2, \ldots, N_{P}$ the mean power

$$
R_{\widetilde{C}}(m)=\frac{1}{D} \sum_{n \in \Lambda_{\widetilde{C}}} R_{n}(m)
$$

associated with $\widetilde{C}$ is computed (here the set $\Lambda_{\widetilde{C}}$ consists of the $D$ values of $n$ that identify the subset of nodes $\left\{a_{n}\right\}$ forming $\widetilde{C}$ );

(4) the "spread" $\sigma_{\widetilde{C}}$ of the set of powers $\left\{R_{\widetilde{C}}(m), m=\right.$ $\left.1,2, \ldots, N_{P}\right\}$ is evaluated as

$$
\sigma_{\widetilde{C}}(m) \triangleq \sqrt{\frac{1}{N_{P}} \sum_{m=1}^{N_{P}}\left(R_{\widetilde{C}}(m)-\mu_{\tilde{C}}\right)^{2}}
$$

where

$$
\mu_{\widetilde{C}} \triangleq \frac{1}{N_{P}} \sum_{m=1}^{N_{P}} R_{\widetilde{C}}(m)
$$

(5) the steps 2-4 are repeated for any possible trial cluster $\widetilde{C}$; at the end of this exhaustive procedure, the cluster $\hat{C}$ minimizing $\sigma_{\widetilde{C}}(8)$ is selected; in other words, the cluster selection strategy, dubbed spread minimization (SM) in the following, can be summarized as

$$
\widehat{C}=\arg \min _{\widetilde{C}} \sigma_{\widetilde{C}}
$$

The rationale behind this technique is to search for a cluster such that the power measurements for its nodes exhibit the minimum spread, that is, the smallest sensitivity to fading; in fact, a small spread indicates that the (deterministic and distance-dependent) path loss term prevails over (stochastic) fading terms in the model expressed by (2).

The main drawbacks of the SM strategy are represented by (a) the need of evaluating of a set of $\left(\begin{array}{c}N_{T} \\ D\end{array}\right)$ distinct metrics $\sigma_{\widetilde{C}}(8)$, where $\left(\begin{array}{c}N_{T} \\ D\end{array}\right)=N_{T} ! /\left(D !\left(N_{T}-D\right) !\right)$ indicates the number of unordered collections of $D$ distinct elements extracted from a set of $N_{T}$; (b) the exhaustive search for the minimum over this set. In fact, these tasks entail a substantial 
computational burden at the anchor. It is important to point out, however, that the task of cluster selection can be carried out without strict real-time constraints (hence, off line) at the anchor, once the whole measurement process has been completed. Moreover, the anchor can be a fixed device connected to the power network, so that, since it is not limited in the energy and/or size, it can be very powerful.

Finally, it is worth noting that

(i) in principle, the size $D$ of the cluster could be optimized considering progressively larger clusters in our search (10), so that the optimal cluster size $N$ could be identified; however, this approach would make the problem computationally unmanageable for large values of $N_{T}$;

(ii) a conceptually similar approach has been adopted in $[4,9]$ to discriminate between LOS and NLOS conditions in a wireless link;

(iii) the performance of the SM strategy is not affected by the presence of correlated shadowing. In fact, in this case, adopting the representation of (3) for the PL, the term $(1 / N) \sum_{n=1}^{N} \operatorname{LSF}_{n}$ in (4) can be rewritten as $\mathrm{LSF}_{\text {mean }}+(1 / N) \sum_{n=1}^{N} \Delta \mathrm{LSF}_{n}$, where $\mathrm{LSF}_{\text {mean }}$ denotes a bias due to the above-mentioned phenomenon. Then, it can be shown that the variability of the right hand side of (7) as well as the spread evaluated according to (8) undergo a reduction with respect to the case of uncorrelated LSF terms. For this reason, the nodes affected by correlated shadowing are likely to be included in the cluster, and, since such nodes are also spatially close, the weight of the terms $\Delta \mathrm{PL}$ and SSF can be appreciably reduced.

3.2. Cluster Selection Based on the Estimation of a Coarse Distance. The second procedure for cluster selection is based on the idea of grouping nodes whose distance from the anchor appears to be close to that evaluated for the target node. This strategy consists of the following steps:

(1) $N_{P}$ independent power measurements $\left\{R_{n}(m), m=\right.$ $\left.1,2, \ldots, N_{P}\right\}$ are acquired by the anchor for the node $a_{n}$ (with $n=1,2, \ldots, N_{T}$ ) reached by a query signal from the anchor;

(2) the anchor processes $R_{n}(m)$, with $m=1,2, \ldots, N_{P}$, to estimate its distance $d_{n}(m)$ from the node $a_{n}$, with $n=1,2, \ldots, N_{T}$ (the adopted estimation technique is illustrated in Sestion 3.3);

(3) the average distance $d_{n}$ of the anchor from $a_{n}$, with $n=1,2, \ldots, N_{T}$, is evaluated as

$$
d_{n}=\frac{1}{N_{P}} \sum_{m=1}^{N_{P}} d_{n}(m)
$$

(4) the anchor accomplishes an exhaustive search to identify, in the set of nodes, the $D$ elements whose distance is closer to the average distance $d_{\mathrm{tg}}$ measured for the target node; formally, this strategy for the selection of the optimal cluster $\hat{C}$ can be expressed, in analogy to (10), as

$$
\hat{C}=\arg \min _{\widetilde{C}} \sum_{n \in \Lambda_{\tilde{C}}}\left|d_{n}-d_{\mathrm{tg}}\right| .
$$

Note, however, that this strategy, called distance estimation (DE) in the following, is substantially simpler than that expressed by (10), since it involves a search over a set of $N_{T}$ nodes, instead of the family of all possible clusters of $D$ nodes.

Finally, it is important to point out that this algorithm does not search for the set of $D$ nodes spatially closer to the target node, but identifies the nodes whose distance is closer to that of the target node $d_{\mathrm{tg}}$. In other words, it selects the nodes closer to a circumference having radius $d_{\mathrm{tg}}$ and the anchor in its center.

3.3. Target Distance Estimation in the Presence of a Cluster of Nodes. It is well known that, in free space propagation, the average power $R_{x}(d)$ (in $\mathrm{dBm}$ ) captured by a receiver placed at a distance $d$ from a transmitter can be expressed as $[15,18]$

$$
R_{x}(d)=P_{0}-10 \xi \log \left(\frac{d}{d_{0}}\right)
$$

where $P_{0}$ represents the power level (in $\mathrm{dBm}$ ) measured at the so-called reference distance $d_{0}$, whereas $\xi$ is the path loss exponent. Note that this expression cannot be exploited as it is for accurately estimating the distance $d$ from a single measurement of average received power, since, as already mentioned in Section 2, the path loss exponent $\xi$ is a random variable. To remove this uncertainty in (13), $\xi$ is replaced by its mean value $\bar{\xi}$ of this parameter in the following. Then, if $R_{x}(d)$ is known, the distance $d$ can be roughly estimated as

$$
d=k \cdot 10^{-R_{x}(d) /(10 \bar{\xi})},
$$

where $k \triangleq d_{0} \cdot 10^{-\left(P_{0} / 10 \bar{\xi}\right)}$.

The expression (14) can be certainly exploited for the same target even in the presence of a cluster of nodes. In fact, an estimate $d_{\text {coop }}$ of the distance of the target node from the anchor can be computed as

$$
d_{\text {coop }}=k \cdot 10^{-P_{\text {av }} /(10 \bar{\xi})}
$$

where

$$
P_{\mathrm{av}} \triangleq \frac{1}{N N_{P}} \sum_{n=1}^{N} \sum_{m=1}^{N_{P}} R_{n}(m)
$$

regardless the adopted cluster selection strategy.

The proposed distance estimation algorithm benefits from spatial diversity [21] and multiuser diversity [22], since $N_{P}$ independent power measurements are acquired for each node of a cluster $\hat{C}$, consisting of $N$ distinct nodes 
(i.e., users). It is also worth pointing out that the number $N_{P}$ of independent measurements that can be acquired from a single node is limited by the availability of independent channel realizations and, consequently, by the characteristics of the considered environment [23]; in addition, the selection of a large value for the cluster size $N$ can lead to a significant residual error $\triangle \mathrm{PL}$ (see (4)). The joint exploitation of the two forms of diversity allows to mitigate the problem of correlated fading [23] and to improve the overall accuracy of the system.

\section{Methods to Acquire Independent RSS Measurements}

Both the SM and DE algorithms for cluster selection rely on the availability of $N_{P}$ independent data for each node. In this Section, we illustrate how such data can be acquired. In particular, two methods are proposed below; one is based on the use of an oscillating beam pattern at a fixed anchor, the other one is based on the use of a frequency sweep.

4.1. Oscillating Beam. The use of an oscillating beam pattern has been adopted in $[23,24]$ to generate a sort of "artificial" time diversity in mobile wireless channels. In our scenario, the implementation of this solution requires the anchor to be equipped with an antenna array fed by phase-controlled signals, so that the main lobe of the radiation pattern can be properly steered. Note that, in a rich scattering environment, different transmission lobes can lead to independent multipath propagation and, hence, to independent channel realizations. In particular, $[23,24]$ evidence that this technique offers the availability of a large number (say, one hundred) of independent channels between two wireless terminals in a static scenario. In our simulations, however, an oscillating beam with 10 distinct angular steps only has been assumed for the reasons illustrated in Section 4.3; this still provides good spatial diversity and, at the same time, does not restrict the significance of the acquired results to a specific propagation environment. Note that (a) when an oscillating beam is used, the index $m$ of $R_{n}(m)$ (see Sestions 3.1 and 3.2) uniquely identifies the orientation of the radiation beam; (b) the use of an oscillating beam increases the complexity of the fixed anchor, but does not affect that of the mobile devices.

4.2. Frequency Sweep. An alternative method to extract independent channel realizations in a static scenario consists of exploiting frequency diversity; in fact, power measurements can be deemed independent if they are collected at multiple frequencies, such that the spacing between adjacent frequencies exceeds the coherence bandwidth of the communication channel [25]. Note that, in this scenario, the index $m$ of $R_{n}(m)$ uniquely identifies the frequency at which the $m$ th power measurement is acquired.

Unluckily, this approach is substantially more complicated than the previous one, since it requires the implementation of mobile nodes with a wideband RF front end.
4.3. Remarks. Even if the methods described above can provide a large number of independent power measurements, only a limited number of such measurements are required by the proposed ranging techniques in a rich scattering indoor environment. For instance, in such a scenario, if the angular step of the beam steering is large, the electromagnetic signals captured along different directions are expected to have travelled along paths characterized by different distances; generally speaking, this does not help to improve the accuracy of our ranging procedure. For this reason, it is recommended to acquire a limited number of independent measurements limiting the deviation of the beam steering, so that small scale fading can be averaged out without appreciably influencing the path loss. Similar considerations apply to the case in which the step size in a frequency sweep is large.

\section{Numerical Results}

This section is organized as follows. First, we comment on the relationship between the distribution of cluster nodes and the achievable accuracy in cluster-based ranging. Then, we analyse various numerical results about the ranging accuracy achieved by the proposed strategies in two different scenarios.

5.1. Spatial Distribution of Cluster Nodes and Ranging Accuracy. When the distances between distinct nodes of a given cluster are not appreciably smaller than the distance between the cluster center and the fixed anchor, the "spread" error $\Delta \mathrm{PL}$ is expected to seriously limit the ranging accuracy achievable by a localization system. To understand the relevance of this problem, let us consider a specific cluster $C$, collecting 5 nodes whose structure is illustrated in Figure 3. In this case, it is assumed that the target is the central node and that, for the sake of simplicity, the distance between the nodes $a(1)$ and $a(2)(a(3)$ and $a(4))$ and the anchor is $r_{1}=r_{2}=r_{0}-\epsilon\left(r_{3}=r_{4}=r_{0}+\epsilon\right)$, where $r_{0}$ is the distance between the target node and the anchor. If the path loss model (1) characterized by an exponential decay [18] is adopted, the power $R$ received by a transmitter located at a distance $d$ from the anchor is given by

$$
R(d)=P_{0}-10 \xi \log _{10}(d)+K,
$$

where $K$ represents the sum of the LSF and SSF contributions. Then, for the $n$th node (with $n=0,1, \ldots, 4$ ) and considering the availability of a single power measurement for each node (i.e., $N_{P}=1$ ) for the sake of simplicity, the anchor exploits the power measurement

$$
R_{n}=P_{0}-10 \xi \log _{10}\left(r_{n}\right)+K_{n}
$$

to compute the mean received power

$$
R_{\mathrm{av}}=\frac{1}{5} \sum_{n=0}^{4} R_{n}=P_{0}-\mathrm{PL}_{\text {mean }}+\frac{1}{5} \sum_{n=0}^{4} K_{n},
$$

where

$$
\mathrm{PL}_{\text {mean }}=\frac{10 \xi \log _{10}\left(r_{0}\right)+20 \xi \log _{10}\left(r_{1}\right)+20 \xi \log _{10}\left(r_{3}\right)}{5} \text {. }
$$




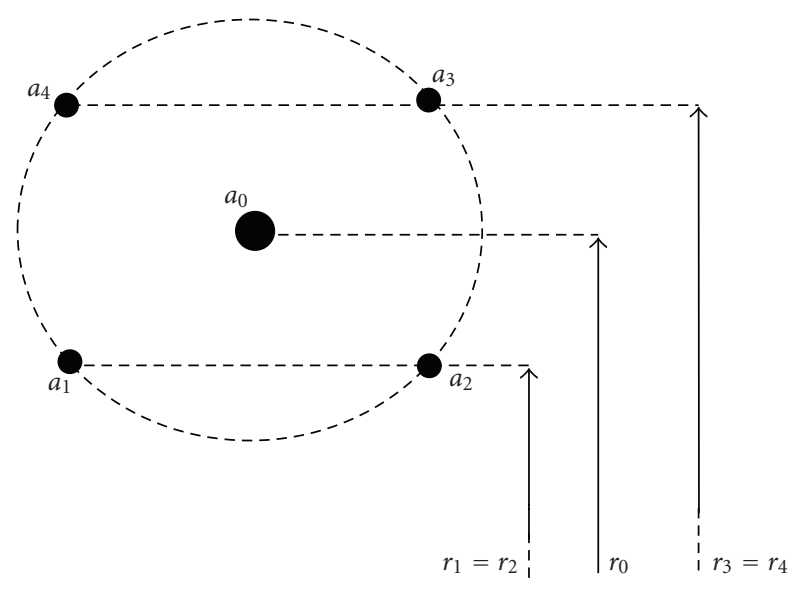

Figure 3: Possible spatial distribution of 5 radiating nodes.

In the last expression, the contribution of the terms $\left\{K_{n}\right\}$ accounts for the fading affecting the nodes; moreover, the path loss $\mathrm{PL}_{\text {mean }}$, depending on the distance between the cluster center and the anchor, can be rewritten as

$$
\begin{aligned}
\mathrm{PL}_{\text {mean }} & =10 \xi \log _{10}\left(r_{0} \cdot r_{1}^{2} \cdot r_{3}^{2}\right)^{1 / 5} \\
& =10 \xi \log _{10}\left(r_{0} \cdot\left(r_{0}-\epsilon\right)^{2} \cdot\left(r_{0}+\epsilon\right)^{2}\right)^{1 / 5} \\
& =10 \xi \log _{10}\left(r_{0}^{5}-2 r_{0}^{3} \epsilon^{2}+r_{0} \epsilon^{4}\right)^{1 / 5},
\end{aligned}
$$

to evidence its dependence on the term $\epsilon$. In any application, we have that $\epsilon \neq 0$ (since the nodes belonging to the cluster associated with a given target cluster do not coincide with the target itself), so that a systematic source of error (i.e., a bias) is found in ranging estimation based on node cooperation. Unfortunately, a theoretical analysis of this problem appears unfeasible, since the nodes of a given cluster are not expected to follow a regular distribution in any indoor application. For this reason, the relevance of the irreducible residual error $\triangle \mathrm{PL}$ (related to the presence of $\epsilon$ in the above-mentioned example) is assessed via computer simulation only in the following.

The problem of the effects, on the achievable performance, of the spatial distribution of the nodes forming a cluster around a given target deserves also the following comments. As stated in Section 2, the aim of the anchor in cluster generation according to the proposed algorithms is to search for the nodes that can minimize the ranging error and not for those forming a dense set. Unluckily, in an indoor scenario characterized by rich scattering, an anchor looking for nodes close to a given target tends to discard all those nodes whose transmission is affected by severe fading, since they appear far from the target location. This explains why the proposed techniques for the generation of clusters take advantage of cooperating nodes which are not close to the target node; this is evidenced by Figure 4, illustrating a possible spatial distribution of a node population and of the cluster nodes selected for a given target.

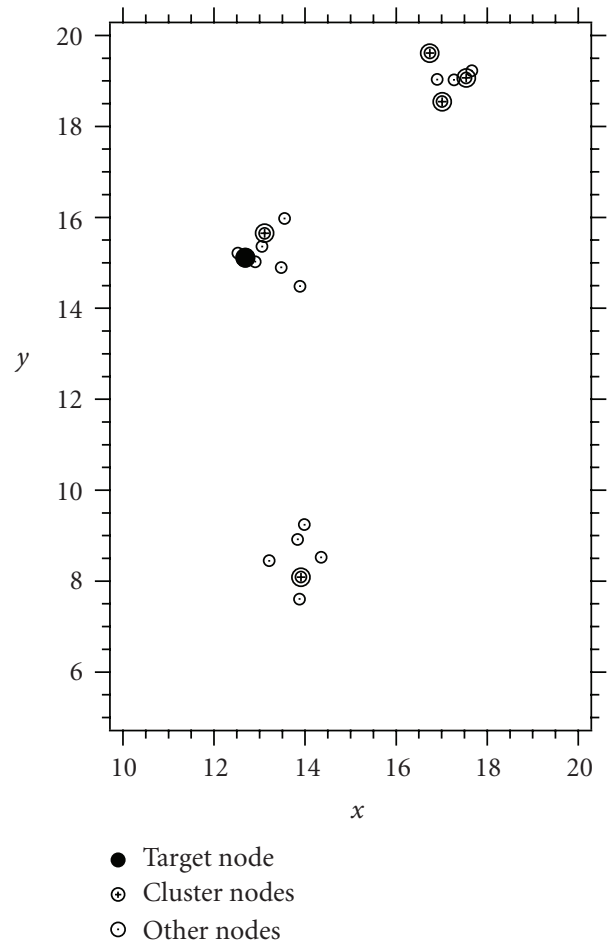

FIGURE 4: Example of spatial node distribution and of a cluster of nodes selected for ranging.

5.2. Ranging Accuracy. The performance offered by the proposed ranging technique has been assessed resorting to computer simulations. In all the simulations, the UWB indoor channel model adopted in $[14,15]$ has been used. This model is characterized by (a) an exponential path loss (see $[14$, equation (12)]) with a Gaussian distributed path gain exponent; (b) a lognormal large-scale fading (see [14, equation (14)]) with zero mean and random variance (a Gaussian distribution is adopted); (c) a Nakagami smallscale fading with the $m$-factor equal to $1 / 2$ (see [15, equation (19)]). All the parameters characterizing this channel model are summarized in [14, Table II]; note that in our simulations, NLOS channel models have been used, when not differently stated.

Two different bidimensional scenarios have been considered for the cluster structure. The first scenario (dubbed scenario \#1 in the following) is characterized by a uniform distribution of nodes and aims at modelling a rich scattering environment in an indoor industrial/commercial open space. In this case, the anchor is placed at a fixed distance of $20 \mathrm{~m}$ from the centre of an area having a square shape with a side $S$ ranging from $3 \mathrm{~cm}$ to $12 \mathrm{~m} ; 20$ nodes are uniformly distributed in such an area, and one is randomly selected as the target. The second scenario (called scenario \#2 in the following) is more suitable to modelling a residential indoor environment (e.g., an office or an hospital) and is characterized by (a) an anchor placed at a fixed distance of $20 \mathrm{~m}$ from the centre of a square region having fixed side equal to $12 \mathrm{~m}$; (b) 20 nodes divided in 3 groups (for instance, each group could consist of the nodes located in a 


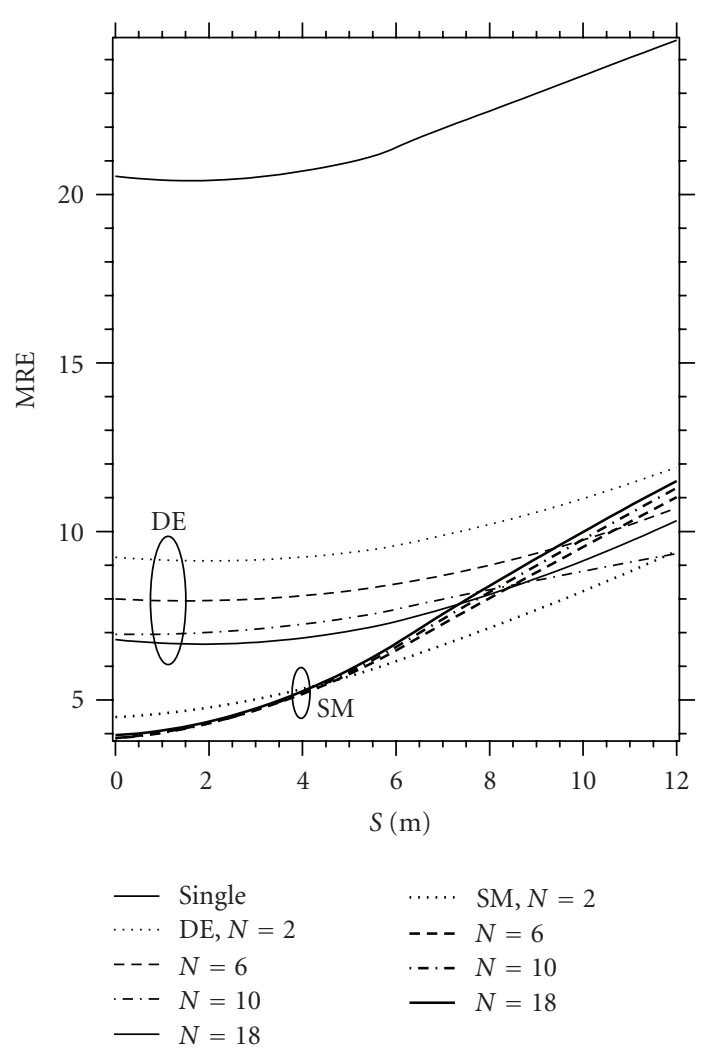

FIGURe 5: Comparison between the MRE provided by the proposed ranging techniques and by a traditional RSS-based technique in the presence of uniformly distributed nodes and NLOS propagation.

different room of the same floor in an indoor environment); (c) the centre of each group is randomly placed within the square region of fixed side and the coordinates of the group nodes are Gaussian distributed around it (the variance of this distribution is properly adjusted to ensure that the side $G$ of the square area containing the group ranges from about $2 \mathrm{~cm}$ to $6 \mathrm{~m}$ ). The target is selected randomly among the nodes. Note that, for both the scenarios, the anchor is always out of the area over which the nodes are distributed.

System performance is assessed in terms of mean ranging error (MRE), defined as the difference between the estimated distance of the target from the anchor and the true distance of the target from the anchor averaged over 500 distinct trials of the ranging procedure. In addition, in the following simulation results, when not differently stated, it is assumed that 10 independent channel realizations are available for each node; we deem this hypothesis realistic, independently of the specific scenario (see Sestion 4.1).

Figure 5 compares the MRE, versus the spatial size $S$ of the area in which the nodes are distributed, of the proposed strategy (using DE or SM for the generation of clusters) with that of a standard single node RSS technique for ranging [12] in scenario \#1. These results show that the former strategy substantially outperforms the latter one, characterized by an MRE comparable to the distance of the target node. The improvement offered by an increase in the number of nodes forming the cluster is evidenced by Figure 6, referring to

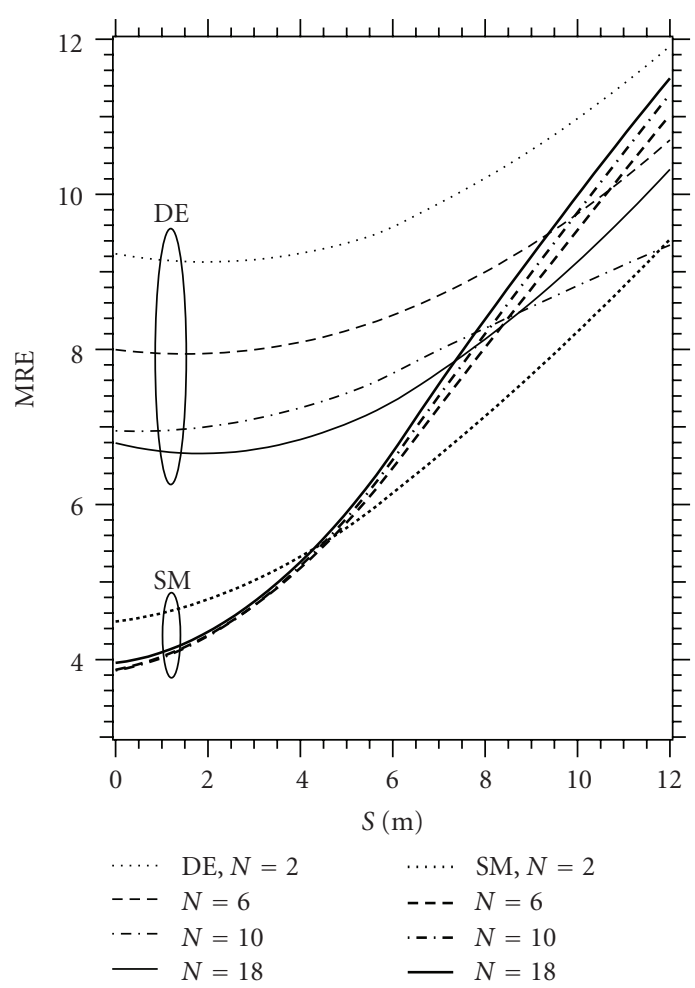

FIGURE 6: MRE provided by the SM and the DE algorithms in the presence of uniformly distributed nodes and NLOS propagation.

the case of the DE and SM techniques only. For instance, if the DE method is adopted, the MRE is limited to about $9 \mathrm{~m}, 8 \mathrm{~m}$, and $7 \mathrm{~m}$ with clusters made of $N=2,6$, and 10 nodes, respectively, note that, when a cluster includes more than 10 nodes, the residual error $\Delta \mathrm{PL}$ can lead to a slight performance degradation. The SM algorithm allows to achieve even better accuracies than the DE; in fact, when $N=2$ nodes form the cluster, the MRE is approximately equal to $4.5 \mathrm{~m}$, whereas the minimum error is reduced to about $4 \mathrm{~m}$ if $N$ is increased to 6 . These results also evidence that SM technique is more sensitive to node density than the DE, which does not appreciably depend on the size of the environment. In fact, if the scenario is densely populated, the SM method outperforms the DE one, whereas the accuracy is comparable when the node distribution is more sparse or even worse when $N>6$. This result can be related to the different rationales behind these two techniques and, in particular, to the fact that the SM aims at mitigating fading effects independently of the residual errors $\left\{\Delta \mathrm{PL}_{n}\right\}$, whereas the DE minimizes the differences in distance measurements, so coping with all the sources of error (residual errors $\left\{\Delta \mathrm{PL}_{n}\right\}$ and fading) indistinctly.

Similar comments can be expressed for the results shown in Figure 7, referring to scenario \#2. Note that, despite the substantial difference between the test scenarios, the performance enhancement provided by the proposed techniques is similar to that achieved in scenario \#1.

Our methods have been also tested in the presence of quasi-LOS propagation conditions, assuming the node 


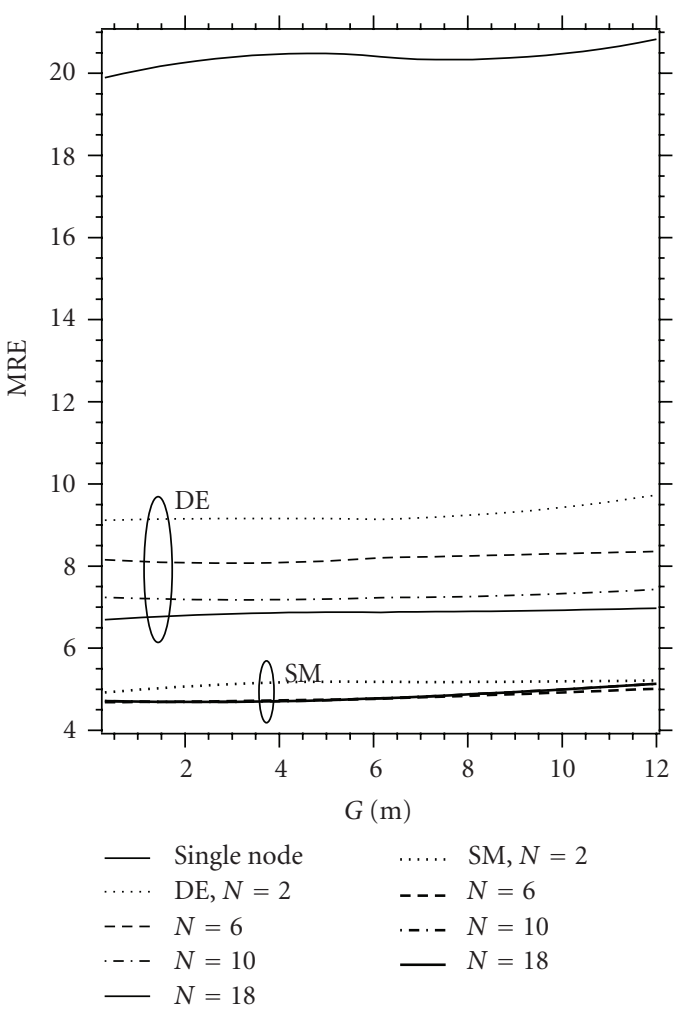

FIGURE 7: Comparison between the MRE provided by the proposed ranging techniques and by a traditional RSS-based technique in the presence of a clustered node distribution and NLOS propagation.

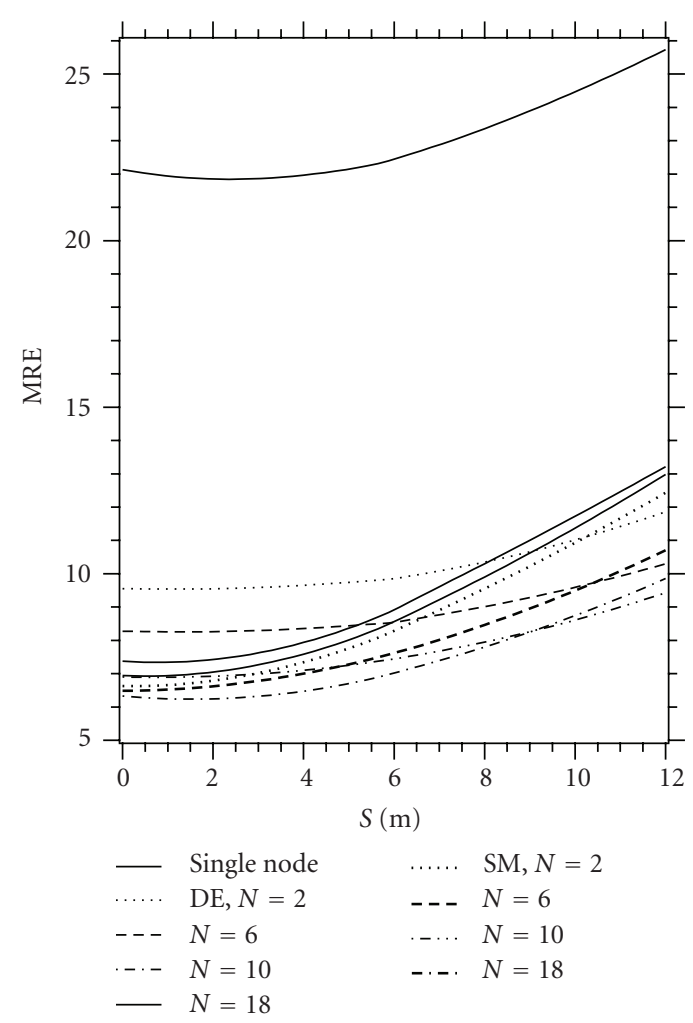

FIgURE 8: Comparison between the MRE offered by the proposed ranging techniques and by a traditional RSS-based technique in the presence of uniformly distributed nodes and quasi-LOS propagation.

(ii) the ranging accuracy provided by the SM and the DE algorithms depends on the overall number of nodes in the environment rather than on their density; in fact these algorithms cooperatively exploit nodes which can be placed far away from the target node, as already discussed in the previous Paragraph,

(iii) further simulations, whose results are not presented here for space limitations, have been run to assess the achievable performance when the generation of clusters is based on a distributed procedure, carried out by the nodes themselves. Our results have evidenced that, since severe fading can lead to uncorrelated channels among the nodes and between the nodes and the anchor, distributed node selection does not provide a substantial improvement, even with the respect to the case of nodes randomly selected by an anchor.

\section{Conclusions}

In this paper, a novel ranging technique based on RSS and suitable to indoor scenarios affected by severe multipath fading has been presented. The technique improves the quality of RSS-based estimation: (a) exploiting the signals radiated by a cluster of nodes; (b) averaging over multiple independent power measurements for each node of the 


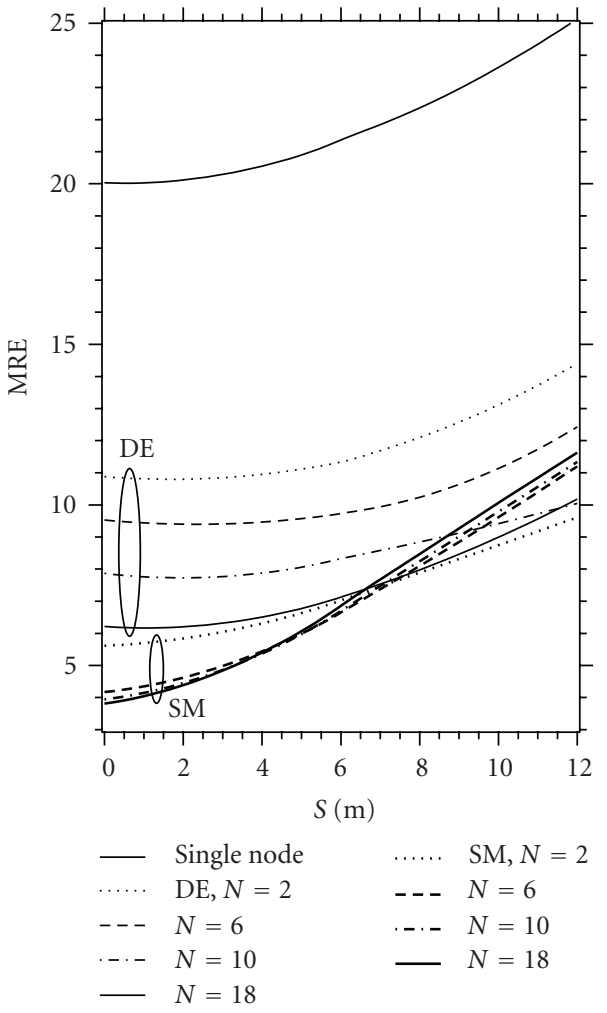

FIgURE 9: Comparison between the MRE offered by the proposed ranging techniques and by a traditional RSS-based technique in the presence of uniformly distributed nodes and NLOS propagation; $N_{P}=5$ independent channel realizations are assumed for each node.

cluster. Specific algorithms for the generation of clusters and for the acquisition of power measurements have been illustrated.

Numerical results have evidenced that the proposed ranging technique is substantially more accurate than traditional strategies in specific scenarios. In addition, thanks to the centralized nature of the algorithms for cluster generation, it allows to move the complexity of an indoor positioning system to a limited number of fixed anchors, so reducing its maintenance costs and making possible to adopt cheap and simple portable wireless nodes. Finally, it is worth mentioning that in poorly populated areas, good performance results can be still achieved if the node density is artificially increased using multiple dummy devices in the environment.

\section{Acknowledgments}

The authors wish to acknowledge the activity of the Network of Excellence in Wireless COMmunications (NEWCOM++, contract no. 216715), supported by the European Commission and that has motivated this work. The authors would like also to thank the anonymous reviewers for their comments which have been extremely helpful in improving the quality of the paper.

\section{References}

[1] http://www.ubisense.net.

[2] N. Patwari, J. N. Ash, S. Kyperountas, A. O. Hero III, R. L. Moses, and N. S. Correal, "Locating the nodes: cooperative localization in wireless sensor networks," IEEE Signal Processing Magazine, vol. 22, no. 4, pp. 54-69, 2005.

[3] T. Roos, P. Myllymaki, and H. Tirri, "A statistical modeling approach to location estimation," IEEE Transactions on Mobile Computing, vol. 1, no. 1, pp. 59-69, 2002.

[4] N. A. Alsindi, K. Pahlavan, B. Alavi, and X. Li, "A novel cooperative localization algorithm for indoor sensor networks," in Proceedings of the IEEE 17th International Symposium on Personal, Indoor and Mobile Radio Communications (PIMRC '06), pp. 1-6, Helsinki, Finland, September 2006.

[5] N. Patwari, A. O. Hero III, M. Perkins, N. S. Correal, and R. J. O'Dea, "Relative location estimation in wireless sensor networks," IEEE Transactions on Signal Processing, vol. 51, no. 8, pp. 2137-2148, 2003.

[6] J.-C. Chen, Y.-C. Wang, C.-S. Maa, and J.-T. Chen, "Networkside mobile position location using factor graphs," IEEE Transactions on Wireless Communications, vol. 5, no. 10, pp. 2696-2704, 2006.

[7] M. Castillo-Effen, W. A. Moreno, M. A. Labrador, and K. P. Valavanis, "Adapting sequential Monte-Carlo estimation to cooperative localization in wireless sensor networks," in Proceedings of the IEEE International Conference on Mobile Ad Hoc and Sensor Sysetems (MASS '06), pp. 656-661, Vancouver, Canada, October 2006.

[8] J. Borras, P. Hatrack, and N. B. Mandayam, "Decision theoretic framework for NLOS identification," in Proceedings of the 48th IEEE Vehicular Technology Conference (VTC '98), vol. 2, pp. 1583-1587, May 1998.

[9] X. Li, K. Pahlavan, D. Ramsburg, and R. Passmore, "Phase I Final Report: Innovative Methods for Geolocation and Communication with UWB Mobile Radio," Internal Report for DARPA, May 2004.

[10] S. Gezici, Z. Tian, G. B. Giannakis et al., "Localization via ultra-wideband radios: a look at positioning aspects of future sensor networks," IEEE Signal Processing Magazine, vol. 22, no. 4, pp. 70-84, 2005.

[11] I. Oppermann, M. Hämäläinen, and J. Iinatti, UWB: Theory and Applications, John Wiley \& Sons, New York, NY, USA, 2004.

[12] T. Gigl, G. J. M. Janssen, V. Dizdarević, K. Witrisal, and Z. Irahhauten, "Analysis of a UWB indoor positioning system based on received signal strength," in Proceedings of the 4th Workshop on Positioning, Navigation and Communication (WPNC '07), pp. 97-101, March 2007.

[13] L. J. Greenstein, S. S. Ghassemzadeh, S.-C. Hong, and V. Tarokh, "Comparison study of UWB indoor channel models," IEEE Transactions on Wireless Communications, vol. 6, no. 1, pp. 128-135, 2007.

[14] A. F. Molisch, "Ultrawideband propagation channels-theory, measurement, and modeling," IEEE Transactions on Vehicular Technology, vol. 54, no. 5, pp. 1528-1545, 2005.

[15] A. F. Molisch, D. Cassioli, and C. C. Chong, "A comprehensive standardized model for ultrawideband propagation channels," IEEE Transactions on Antennas and Propagation, vol. 54, no. 11, pp. 3151-3166, 2006.

[16] J. Romme and B. Kull, "On the relation between bandwidth and robustness of indoor UWB communication," in Proceedings of the IEEE Conference on Ultra Wideband Systems and Technologies, pp. 255-259, November 2003. 
[17] N. A. Alsindi, K. Pahlavan, and B. Alavi, "An error propagation aware algorithm for precise cooperative indoor localization," in Proceedings of the Military Communications Conference (MILCOM '06), pp. 1-7, Washington, DC, USA, October 2006.

[18] T. S. Rappaport, Wireless Communications: Principles and Practice, Prentice Hall, Upper Saddle River, NJ, USA, 2nd edition, 2002.

[19] A. Papoulis and S. U. Pillai, Probability, Random Variables and Stochastic Processes, McGraw-Hill, New York, NY, USA, 4th edition, 2001.

[20] P. Krishna and D. Husak, "RFID infrastructure," IEEE Communications Magazine, vol. 45, no. 9, pp. 4-10, 2007.

[21] S. N. Diggavi, N. Al-Dhahir, A. Stamoulis, and A. R. Calderbank, "Great expectations: the value of spatial diversity in wireless networks," Proceedings of the IEEE, vol. 92, no. 2, pp. 219-270, 2004.

[22] A. Nosratinia, T. E. Hunter, and A. Hedayat, "Cooperative communication in wireless networks," IEEE Communications Magazine, vol. 42, no. 10, pp. 74-80, 2004.

[23] S. A. Zekavat and C. R. Nassar, "Transmit diversity via oscillating-beam-pattern adaptive antennas: an evaluation using geometric-based stochastic circular-scenario channel modeling," IEEE Transactions on Wireless Communications, vol. 3, no. 4, pp. 1134-1141, 2004.

[24] S. A. Zekavat, C. R. Nassar, and S. Shattil, "Smart antenna spatial sweeping for combined directionality and transmit diversity," Journal of Communications and Networks, vol. 2, no. 4, pp. 325-330, 2000.

[25] J. G. Proakis, Digital Communications, Mcgraw Hill Series in Electrical and Computer Engineering, McGraw-Hill, New York, NY, USA, 4th edition, 2001. 

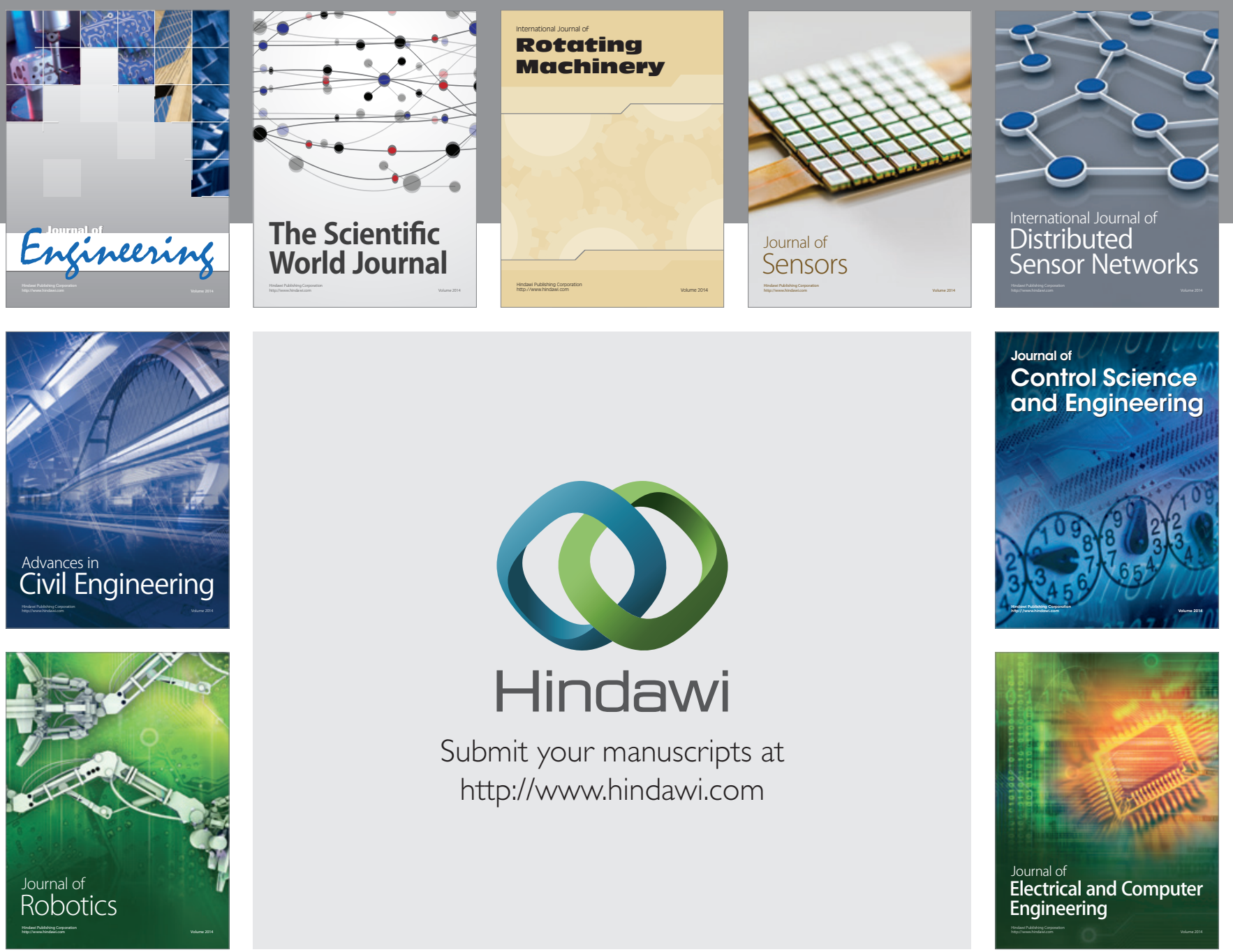

Submit your manuscripts at

http://www.hindawi.com
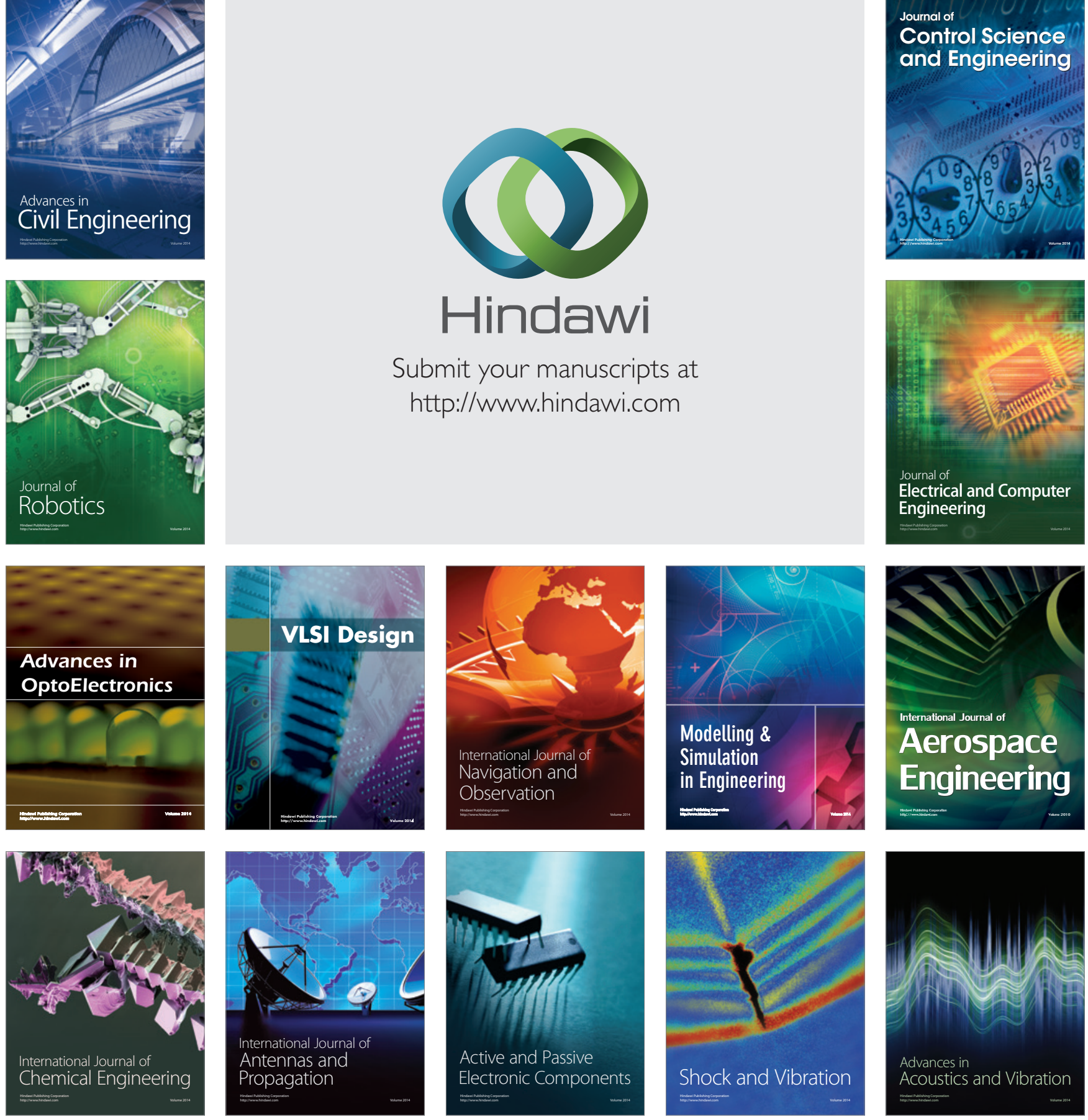